\title{
Traffic Radar: A Holonic Traffic Coordination System Using PROSA++ and D-MAS
}

\author{
Johan Philips, Bart Saint Germain, Jan Van Belle, and Paul Valckenaers \\ Department of Mechanical Engineering, KU Leuven, \\ Celestijnenlaan 300B, 3001 Leuven, Belgium \\ \{first.last\}@mech.kuleuven.be \\ http://www.mech.kuleuven.be
}

\begin{abstract}
This paper presents Traffic Radar, a holonic traffic coordination system focusing on the distributed and dynamic nature of traffic systems. Two main architectural assets enable distributed real-time coordination, the holonic PROSA + + architecture and the delegate multi-agent system (D-MAS) pattern. Well proven and accurate firstorder traffic models are used to model local traffic behaviour. Link and node holons encapsulate local traffic models and offer services to other holons and D-MAS ants in the environment. Early experiments with the Traffic Radar platform show its ability to forecast traffic flows and densities based on individual user intentions. Moreover, they show the ability to explore different routing solutions incorporating traffic density forecasts.
\end{abstract}

Keywords: Holonic system, traffic models.

\section{Introduction}

A traffic system comprises a set of autonomous entities. All entities have, to some extent, a degree of freedom: (1) vehicle drivers have the freedom to select their personal route and timing; (2) traffic infrastructure cannot be centralised entirely. Still it is opportune to coordinate these selfish users. This is mainly achieved by providing information and incentives, such as personal guidance or cost estimation.

The Traffic Radar is a holonic traffic coordination system which creates and maintains a short-term forecast of traffic flows and densities. The short-term forecast is characterised by its high fidelity through a high participation of individual traffic users who propagate their intentions. It provides a unique possibility to explore different routings and timings. The explored alternatives are the key information to guide and steer users towards a system optimum.

Traffic is also characterised by its fluctuating state. Disturbances like car accidents, weather conditions or changing traffic demand influence heavily the traffic flow and density.

The Traffic Radar's holonic architecture is a natural mapping of the traffic infrastructure and its users. The choice of this holonic architecture is further elaborated in the following section.

V. Mařík, J.L. Martinez Lastra, P. Skobelev (Eds.): HoloMAS 2013, LNAI 8062, pp. 163-174, 2013. (C) Springer-Verlag Berlin Heidelberg 2013 


\subsection{Holonic Architecture in Traffic Context}

Two main architectural assets enable distributed real-time coordination, the holonic PROSA + + architecture and the delegate multi-agent system (D-MAS) pattern. In the context of traffic, a holon is defined as an autonomous and cooperative building block of a traffic control system for informing and guiding traffic entities.

Using a holonic architecture requires an identification of different holon types. Responsibilities need to be assigned to each holon type and relationships between holons need to be defined clearly. PROSA ++ is a reference architecture for holonic architectures that reduces impact of changes in decision making by separating concerns. This reference architecture allows to [1]1:

- Separate resource (traffic infrastructure) aspects from product (trip planning and driving to its destination) specific aspects. Typical for traffic control is the difference in goal between selfish traffic users and traffic infrastructure as system-wide optimiser.

- Separate necessary modules, which are generic, from optional modules, which can be domain specific. Different holons in PROSA ++ all hide specific technical details from each other.

- Separate structural aspects of the architecture from algorithmic aspects. Existing scheduling and planning algorithms can be integrated without affecting the basic architecture.

Traffic is characterised by its distributed and dynamic nature. A holon needs to be capable of making decisions that are adapted to events happening in the environment, i.e. the traffic network. The delegate MAS pattern allows holons to exploit detailed and up-to-date information in this environment and to adapt their intentions to these events.

\section{PROSA ++ for Traffic Coordination}

The holonic architecture, PROSA++, is adopted from a well-studied and widely used architecture in manufacturing control systems, PROSA [1]. The PROSA architecture was originally developed for the manufacturing domain. It has also been applied in other application domains (railway systems [3, logistic systems 10, robotic systems [7, and others [9]). This architecture is further elaborated towards PROSA ++ which appeared to be well suited for traffic control systems.

\subsection{PROSA + + Reference Architecture}

The PROSA ++ architecture identifies four holon types as indicated in Figure 1. A distinction is made between products and resources. Products relate to any activity in the world of interest, in this case traffic users. Resources relate to 
enabling entities in the world of interest, in this case traffic infrastructure entities, e.g., links and nodes.

Note that due to the origin of PROSA++ (manufacturing), the terminology product and resource may seem confusing. Nevertheless, their concepts remain valid and have been proven outside the manufacturing domain [9].

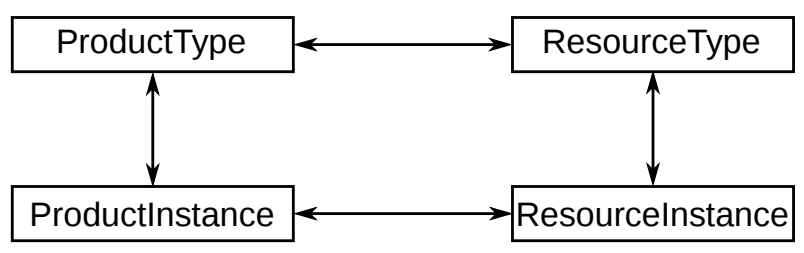

Fig. 1. Holonic reference architecture PROSA ++, based on well-studied and widely used architecture in manufacturing control systems, PROSA [1]

The ResourceInstance holon reflects a specific part of the traffic infrastructure. This holon contains information about the physical entity it reflects, present and future states and what-if functionality. We consider two classes: route infrastructure, i.e. nodes and links, and multi-modal transport, such as trams and buses.

The class information is encapsulated into the ResourceInstance holon. The same interface is used to the remaining system. Preferences to use one or another ResourceInstance holon are expressed in terms of capability (e.g. amount of luggage), availability and trust (reputation from one user towards a ResourceInstance holon).

A ResourceType holon holds policies regarding a specific resource entity or a group of resources. Examples of these policies are: maximum and minimum speed on a link or the presence of bus lanes. Policies are communicated to ResourceInstance holons in order to ensure a correct and desired behaviour on the traffic network entity.

The ProductInstance holon corresponds to a request for a trip in the traffic network, originating from a traffic user. This holon is responsible for planning and guiding the assigned trip correctly and on time. The Product Instance holon searches and evaluates candidate routes and potential multi-modal alternatives. A selection of candidates is presented to the user while the final selection - the intention - is the responsibility of the user. The ProductInstance holon reflects this decision to other holons.

ProductType holons hold all policies regarding a traffic user or a group of users. These policies can be various, some examples:

- Preferences regarding multi-modal transport, vehicle availability.

- Preferences regarding distance.

- Preferences regarding route types (e.g. scenic route), stops allowed or preferred.

These policies are exchanged with the ProductInstance holon to guide the search for good journeys. 


\section{D-MAS for Traffic Coordination}

Delegate multi-agent system (D-MAS) is an architectural pattern that allows a holon to delegate a responsibility to a swarm of lightweight ant agents to support this holon in fulfilling its functions 611. The issuing holon can delegate multiple responsibilities, each of them applying the delegate MAS pattern. The holon may use a combination of delegate multi-agent systems to handle a single responsibility. The D-MAS may also provide services to other holons.

The $D$-MAS pattern translates insights from the food foraging behaviour in ant colonies into the software design [8].

- Refresh-and-evaporate: ants deposit pheromone trails that evaporate unless refreshed by ants walking along such a trail. This translates into: All information in the traffic management system that is subject to real-world dynamics has a finite lifespan. For instance, trip reservations need to be reconfirmed regularly or they are discarded by the resource (e.g. parking space) concerned.

- The environment contributes to the solution: ants deposit their pheromone trails on the real-world environment, allowing them to cope with almost any geometrical complexity by means of a single simple procedure. Translating this mechanism, an environment is created that mirrors the world-of-interest in software (physical traffic infrastructure), and environment components will be made intelligent/cognitive as needed or opportune.

- Swarming: from simple ant behaviours emerges sophisticated colony behaviour. However, the colony needs numerous cheap ants to achieve this. In the model, traffic users create swarms of lightweight agents - called ant agents - that travel virtually across the environment. Because they are virtual entities, ant agents are cheap and can be numerous. These swarms, performing services on behalf of holons, are called a D-MAS.

- Computational efficiency: our ant-like design has a low-polynomial computational complexity in function of the effort needed for the primitive actions of virtual travel through the environment.

\section{Traffic Models}

This section explains briefly the traffic models used by link and node holons to predict traffic flow. Local traffic models comply with simplified kinematic wave theory used in link transmission models 2413. The fixed point formulation of the link transmission model 2413] enables an iterative approach and justifies the usage in a distributed and real-time context.

In the link transmission model individual vehicles are aggregated into a continuous vehicle flow (represented by cumulative vehicle numbers). Figure 2 depicts a schematic representation of a link by the cumulative flow at the beginning of the link (upstream) and at the end of the link (downstream). The cumulative flow at the end of the link is shifted in time as it takes time to travel though 


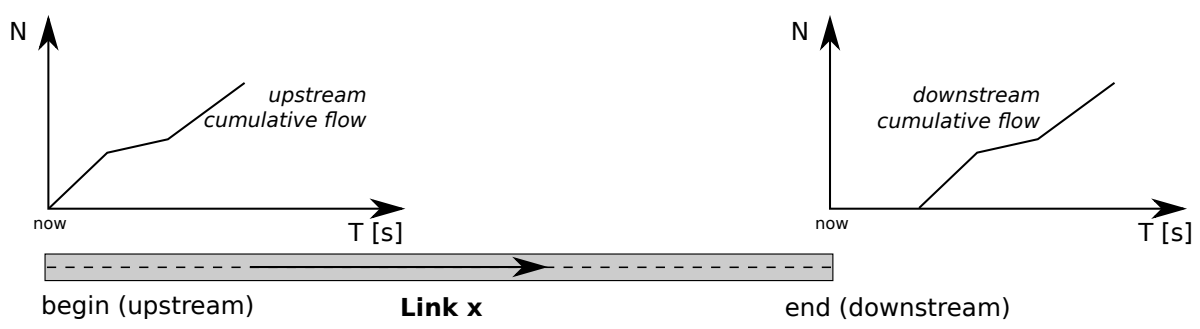

Fig. 2. Representation of a link by begin and end cumulative flow

the link. These cumulative flows are calculated iteratively and are adjusted whenever the context changes or new constraints are appearing from changed traffic demand or traffic supply, e.g. traffic jam and queue spill back due to insufficient downstream capacity or an accident.
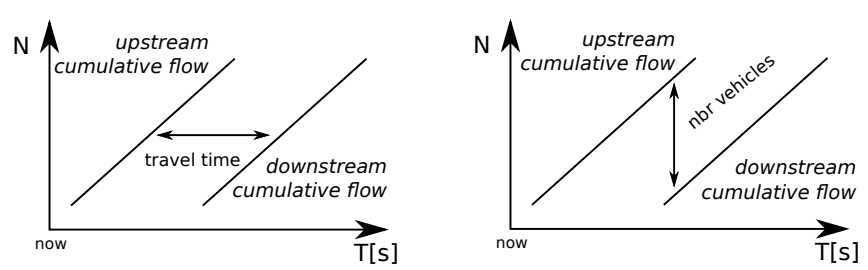

Fig. 3. Properties of link representation by cumulative flows

The representation by cumulative vehicle numbers holds some interesting basic properties: link travel time and total number of vehicles on the link (figure 3). The horizontal distance between a same cumulative count at the beginning of the link and at the end of the link indicates the travel time for the time at which the entrance cumulative count is reached. The vertical distance between begin and end cumulative flow indicates total number of vehicles at that time.

\subsection{Forward Propagation}

This model propagates traffic flow from upstream to downstream within one link. Moreover, the model calculates downstream cumulative flow by adding the appropriate travel time (valid at that point in time) to each point in the upstream cumulative flow. In case there are no previous iterations, free flow travel time is used, i.e. the time to cross the link at maximum speed. If there was a previous iteration, travel time is calculated by comparing up- and downstream cumulative flow from the previous iteration as explained in section 4.

Figure 4 shows forward propagation of a changed upstream cumulative flow in iteration $\mathrm{i}+1$. Travel times are adopted from iteration $\mathrm{i}$ and result in an updated downstream cumulative flow. 


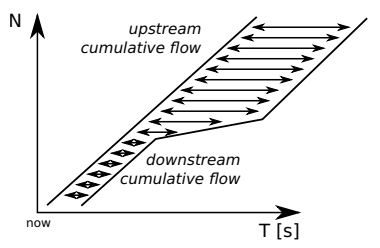

(a) iteration i

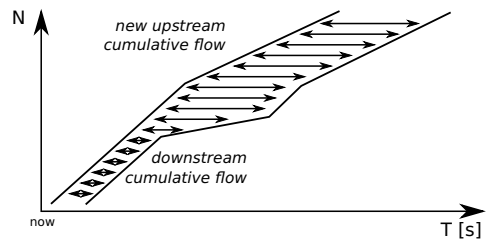

(b) iteration $\mathrm{i}+1$

Fig. 4. Forward propagation

\subsection{Backward Propagation}

Here, the model propagates traffic flow from downstream to upstream within one link. Two steps can be identified in this model: upstream constraint calculation and applying this constraint to the original upstream cumulative flow.

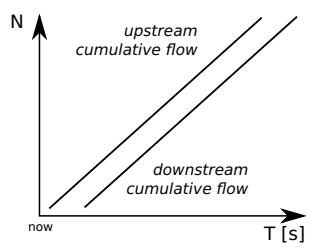

(a)

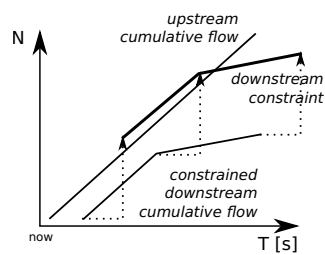

(c)

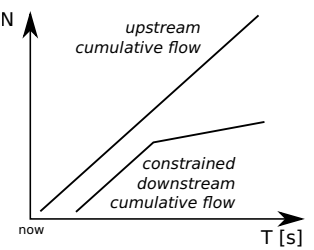

(b)

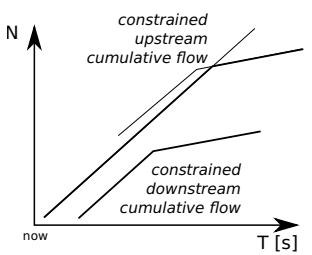

(d)

Fig. 5. Backward propagation

This section explains the model based on an scenario (Figure 5). Please refer to [13] for a full understanding of this model.

The scenario consists of four steps:

1. The link is in free flow state: downstream cumulative flow is shifted with free flow time as compared to the upstream cumulative flow (figure [5]a);

2. A car accident on a downstream link gives rise to a constrained downstream cumulative flow (figure $5 \mathrm{~b}$ );

3. The updated downstream cumulative flow results in an upstream constraint (figure [5]c). This constraint represents the maximum flow which can enter at the beginning of the link. The maximum entrance flow is calculated by 
shifting the upstream cumulative with the spill back time (i.e. the minimum time a queue at the end of the link needs to reach the beginning of the link) and augmented by the numbers of cars at the link in case of traffic jam;

4. The original upstream cumulative flow is constrained by the maximum flow defined by the propagated downstream flow (figure 5 d). The new upstream cumulative flow is formed by the minimum of the original cumulative flow and the maximum flow evaluated in all points.

\subsection{Capacity Constraint}

A node or link has a limited capacity. This corresponds to a maximum gradient in cumulative flows. This maximum gradient is applied to the cumulative flow by lowering the flow to the maximum capacity if the original flow is too high. Figure 6 shows the original cumulative flow and the constrained cumulative. Flow from time $\mathrm{t} 1$ until $\mathrm{t} 2$ is below the maximum capacity and does not need to be constrained. After time t2 flow gets higher and is constrained accordingly until the intersection from the gradient with the original cumulative flow: $t 4$. This procedure is repeated until the full cumulative flow is constrained.

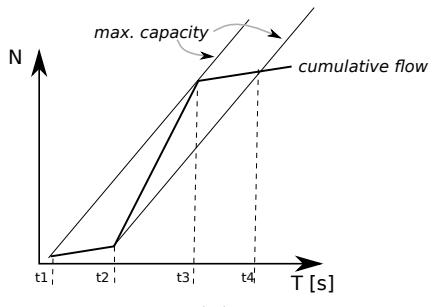

(a)

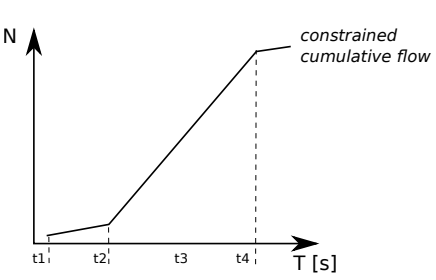

(b)

Fig. 6. Flow constrained by capacity

\section{Implementation: Traffic Radar}

The holonic concepts provided by PROSA ++ and D-MAS have been applied to traffic coordination. This has led to the implementation of the Traffic Radar, which uses PROSA++ to model the traffic infrastructure and several D-MASs to forecast traffic flows.

\section{$5.1 \quad$ Erlang}

Erlang provides massively parallel and distributed processing. Benchmark tests with up to one million processes ensure the creation of large traffic networks with many ants dropping pheromones on links and nodes. Moreover, every erlang process is an actor. Many ideas in the actor model [5] are now applied in multiagent systems [12]. Erlang, therefore, is excellently suited for holonic multi-agent systems and complies with the dynamic and distributed nature of traffic. 


\subsection{Overview}

In the holonic architecture, the traffic infrastructure, i.e. the network, is represented by a set of two distinct ResourceType holons: Link holons and Node holons. Furthermore Vehicle holons, represented by ProductInstance holons, correspond to trip requests in the traffic network, originating from traffic users. Table 1 provides an overview of the different holons and their responsibilities.

Table 1. Overview of the different holons in the Traffic Radar application, their life cycles and services and which D-MAS they use

\begin{tabular}{|l||l|l|l|}
\hline Holon & Life cycle & Services & D-MAS \\
\hline Link & $\begin{array}{l}\text { update state } \\
\text { forward flow propagation } \\
\text { backward queue propagation }\end{array}$ & $\begin{array}{l}\text { execute scenario } \\
\text { proclaim scenario } \\
\text { propagate scenario }\end{array}$ & none \\
\hline Node & detect capacity constraints & $\begin{array}{l}\text { execute scenario } \\
\text { proclaim scenario } \\
\text { propagate scenario }\end{array}$ & flow D-MAS \\
\hline Vehicle & $\begin{array}{l}\text { find solutions } \\
\text { select intention (manual) }\end{array}$ & $\begin{array}{l}\text { exploring D-MAS } \\
\text { select intention D-MAS }\end{array}$ \\
\hline
\end{tabular}

\subsection{Link Holon}

The life cycle of a Link holon is characterised by three distinct actions. Firstly, dynamic map information, received by e.g. a traffic monitoring system, is used to update its state. Secondly, based on this dynamic map information, such as traffic density, propagation of flows and cumulative functions are calculated. Thirdly, back propagation of queues, e.g. due to bottlenecks, is performed.

Link holons also provide services to other holons. The ExecuteScenario service can be used to perform a what-if scenario on the holon. The service returns travel time on the respective link given an arrival time and estimated traffic density. The ProclaimScenario service enables holons to calculate the travel time and save their intention indicating at what time they would arrive and depart on the link. Finally, the PropagateScenario service offers the ability to adapt upstream or downstream flow on the link. Consequently, adapted flow is propagated within the link. Adapting upstream flow results in forward propagation, while downstream flow adaptations result in backward propagation.

\subsection{Node Holon}

The life cycle of a Node holon consists of detecting capacity constraints. If flow inconsistency between upstream and downstream links in a particular node occurs, e.g. due to an accident or structural bottleneck, flow has to be adapted. To propagate traffic flow constraints both upstream and downstream, the Node holon creates flow ants. This propagation models spill back over links and nodes.

The services offered by Node holons are identical to those of Link holons. 


\subsection{Vehicle Holon}

Vehicle holons represent users driving through the traffic network and, therefore, also virtually move through the Link and Node holons. To indicate where a Vehicle holon resides and where it is moving to, it sends out two different D-MASs: exploring D-MAS and intention D-MAS. These D-MASs drop pheromones on the traffic infrastructure holons to respectively search and proclaim the route of the relevant Vehicle holon from its origin to destination.

The life cycle of this holon also contains, besides exploring and intention ant creation, functionality to update its state, containing information such as its origin and destination, the current link or node it resides on, and vehicle properties. If configured so, it also automatically selects a route out of solutions, provided by the exploring ants.

The Vehicle holon offers two services to other holons or external users. Firstly, the set of routing solutions, from its origin to destination, can be requested. Secondly, a user can manually select one of these solutions to commit to. In this case the automatic intention selection is disabled.

\subsection{D-MAS Implementation}

All ants in the different D-MAS have the same life cycle: (1) a scenario is created based on their current state, (2) this scenario is executed on the resource, on which the ant currently resides, and (3) this process is repeated until an end condition has reached. Both scenario creation and execution are D-MAS specific.

In Erlang, these steps are represented by functions, which can be passed to the ant state at creation, reducing the life cycle code to a few lines, as depicted in Listing [1.1]

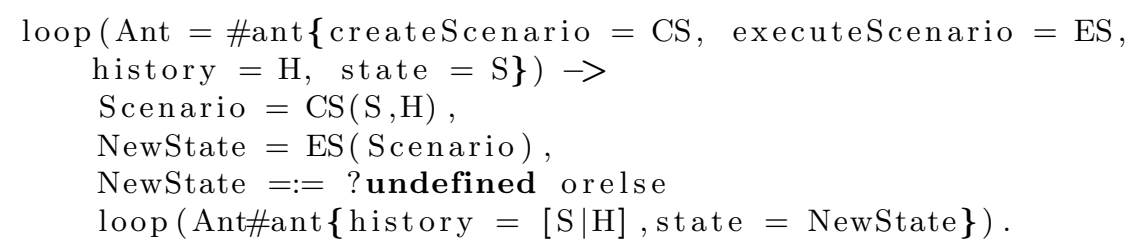

Listing 1.1. Erlang code for generic ant life cycle

\subsection{Exploring D-MAS}

The exploring D-MAS, used by Vehicle holons, move through traffic infrastructure holons to search for a routing solution from the vehicle's origin to destination. On each holon they visit, the ExecuteScenario service is executed to determine travel time on that holon, based on provided arrival time, which is typically in the near future, and the traffic at that time. This service uses the travel time model discussed in section 4 


\subsection{Intention D-MAS}

Vehicle holons also deploy intention ants through the traffic network. These ants receive a selected solution, chosen by their creator, from the solutions gathered by the exploring ants. The intention ants drop pheromones on the relevant holons to proclaim the Vehicle holon's presence on these holons at a certain time. To accomplish this, intention ants employ the ProclaimScenario service of the currently occupied holon. This service determines travel time, similarly to the ExecuteScenario service, based on the travel time model.

The difference with exploring ants is twofold: (1) a solution, i.e. a route, is provided at creation and (2) pheromones are dropped on traffic infrastructure holons to indicate when the vehicle would be where.

\subsection{Flow D-MAS}

The last D-MAS ants, used in the Traffic Radar application, are created by Node holons. As discussed, flow ant creation is triggered by flow inconsistencies in a node. Flow ants make use of the PropagateScenario to adapt upstream or downstream flow. By executing this scenario, a new constraint can exist, in which case the flow ant continues to move upstream or downstream. If no new constraint is formed, the flow ant stops propagating.

\section{Experiments}

This section presents early experiments with the Traffic Radar platform. The experiments show the ability to forecast traffic flows and densities based on individual user intentions.

If the accumulated traffic on a link remains below the link's maximum capacity, the flow is not constrained and a free flow model is used to calculate the cumulative at the end of the link. Figure 7 depicts both cumulative flow at the beginning and end of the link. In this case, no constraints are applied, resulting in free flow. The cumulative function is just shifted into the time.

In contrast, Figure 8 shows an example, in which too many vehicles enter a link and constraints are propagated. This process consists of three propagation waves:

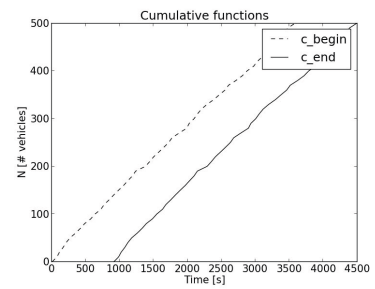

Fig. 7. Flow not constrained by downstream link 


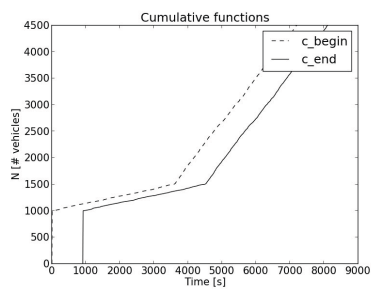

(a) Forward propagation

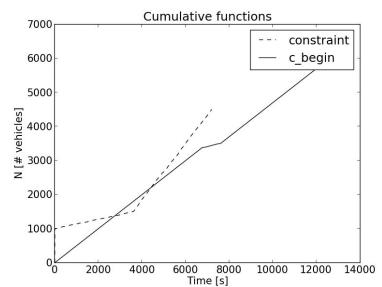

(b) Backward propagation

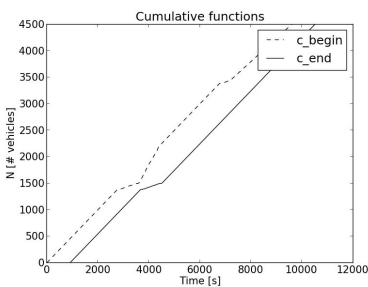

(c) Forward propagation

Fig. 8. Cumulative functions of time versus number of vehicles. These figures illustrate how forward and backward propagation combined with constraint detection enable traffic flow forecasts.

1. Flow is propagated forward, see Figure 8(a), to calculate a cumulative function at the end of the link.

2. Maximum capacities are incorporated, queues are calculated and propagated backward, resulting in a new cumulative function, see Figure 8(b), at the beginning of the link.

3. The new cumulative function is propagated forward once more to obtain the new cumulative function, see Figure $8(\mathrm{c})$, at the end of the link.

\section{Conclusion and Future Work}

In context of the EU FP7 MODUM project, the Traffic Radar is part of a larger infrastructure. On the one hand, real-time traffic data is captured and provided, while on the other hand user requests are provided through various interfaces. The current Traffic Radar platform enables the generation and maintenance of short-term forecasts. This short-term forecasts provide information about the traffic flow and density on links and nodes and can be consulted by both exploring and intention D-MAS. This platform will be tested on a big urban traffic network in the short future. In that stage, the full impact of this novel platform will be measured both in simulation and in a smaller scale in real life.

Acknowledgments. This paper reflects the work funded by European project MODUM (FP7-288205, Models for Optimising Dynamic Urban Mobility).

\section{References}

1. Van Brussel, H., Wyns, J., Valckenaers, P., Bongaerts, L., Peeters, P.: Reference architecture for holonic manufacturing systems: Prosa. Computers in Industry 37(3), 255-274 (1998)

2. Daganzo, C.F.: A variational formulation of kinematic waves: Solution methods. Transportation Research Part B: Methodological 39(10), 934-950 (2005) 
3. De Swert, K., Valckenaers, P., Saint Germain, B., Verstraete, P., Hadeli, H., Van Brussel, H.: Coordination and control for railroad networks inspired by manufacturing control. In: Proceedings of the IEEE Workshop on Distributed Intelligent Systems: Collective Intelligence and its Applications, DIS 2006, pp. 201-206. IEEE Computer Society, Washington, DC (2006)

4. Gentile, G., Meschini, L., Papola, N.: Spillback congestion in dynamic traffic assignment: A macroscopic flow model with time-varying bottlenecks. Transportation Research Part B: Methodological 41(10), 1114-1138 (2007)

5. Hewitt, C.: Viewing control structures as patterns of passing messages. Artificial Intelligence 8(3), 323-364 (1977)

6. Holvoet, T., Weyns, D., Valckenaers, P.: Patterns of delegate mas. In: Proceedings of the 2009 Third IEEE International Conference on Self-Adaptive and SelfOrganizing Systems, SASO 2009, pp. 1-9. IEEE Computer Society, Washington, DC (2009)

7. Philips, J., Valckenaers, P., Aertbeliën, E., Van Belle, J., Saint Germain, B., Bruyninckx, H., Van Brussel, H.: PROSA and delegate MAS in robotics. In: Mařík, V., Vrba, P., Leitão, P. (eds.) HoloMAS 2011. LNCS, vol. 6867, pp. 195-204. Springer, Heidelberg (2011)

8. Valckenaers, P., Van Brussel, H.: Holonic manufacturing execution systems. CIRP Annals - Manufacturing Technology 54(1), 427-432 (2005)

9. Van Belle, J., Philips, J., Ali, O., Saint Germain, B., Van Brussel, H., Valckenaers, P.: A service-oriented approach for holonic manufacturing control and beyond. In: Borangiu, T., Thomas, A., Trentesaux, D. (eds.) Service Orientation in Holonic and Multi-Agent Manufacturing Control. SCI, vol. 402, pp. 1-20. Springer, Heidelberg (2012)

10. Van Belle, J., Saint Germain, B., Valckenaers, P., Van Brussel, H., Bahtiar, R., Cattrysse, D.: Intelligent products in the supply chain are merging logistic and manufacturing operations. In: Proceedings of the 18th IFAC World Congress (August 2011)

11. Verstraete, P., Valckenaers, P., Van Brussel, H., Saint Germain, B., Hadeli, K., Van Belle, J.: Towards robust and efficient planning execution. Eng. Appl. Artif. Intell. 21, 304-314 (2008)

12. Woolridge, M.: Introduction to Multiagent Systems. John Wiley \& Sons, Inc., New York (2009)

13. Yperman, I.: The Link Transmission Model for Dynamic Network Loading. PhD thesis, KU Leuven (2007) 